\title{
Congruency effects between auditory and tactile motion: Extending the phenomenon of cross-modal dynamic capture
}

\author{
SALVADOR SOTO-FARACO \\ Universitat de Barcelona, Barcelona, Spain \\ CHARLES SPENCE \\ University of Oxford, Oxford, England \\ and \\ ALAN KINGSTONE \\ University of British Columbia, Vancouver, British Columbia, Canada
}

\begin{abstract}
Behavioral studies of multisensory integration in motion perception have focused on the particular case of visual and auditory signals. Here, we addressed a new case: audition and touch. In Experiment 1 , we tested the effects of an apparent motion stream presented in an irrelevant modality (audition or touch) on the perception of apparent motion streams in the other modality (touch or audition, respectively). We found significant congruency effects (lower performance when the direction of motion in the irrelevant modality was incongruent with the direction of the target) for the two possible modality combinations. This congruency effect was asymmetrical, with tactile motion distractors having a stronger influence on auditory motion perception than vice versa. In Experiment 2, we used auditory motion targets and tactile motion distractors while participants adopted one of two possible postures: arms uncrossed or arms crossed. The effects of tactile motion on auditory motion judgments were replicated in the arms-uncrossed posture, but they dissipated in the arms-crossed posture. The implications of these results are discussed in light of current findings regarding the representation of tactile and auditory space.
\end{abstract}

The ventriloquism illusion provides a compelling illustration of how information presented in one sensory modality can influence information processing in another sensory modality (e.g., see Howard \& Templeton, 1966). In the laboratory, this illusion is normally stripped down to the presentation of static sounds combined with synchronized light flashes separated spatially from the sounds. Typically, the sounds are mislocalized toward the position of the visual events (see Bertelson, 1999, and Bertelson \& de Gelder, 2004, for recent reviews). However, one of the real-world aspects often lost in the typical laboratory study of the ventriloquism illusion is an appreciation of the role that stimulus movement might play in modulating multisensory integration. Despite isolated studies in the past (e.g., Allen \& Kolers, 1981; Ma-

S.S.-F. received funding from the McDonnell-Pew Center for Cognitive Neuroscience in Oxford and the Killam Trust (Canada). A.K. received support from the Michael Smith Foundation for Health Research, the Natural Sciences and Engineering Research Council of Canada, and the Human Frontier Science Program. We thank Jessica Lyons for help in running the experiments. Correspondence concerning this article should be addressed to S. Soto-Faraco, Parc CientificUniversitat de Barcelona, Barcelona, Spain (e-mail: ssoto@psico.psi. ub.es). teeff, Hohnsbein, \& Noack, 1985; Staal \& Donderi, 1983; Urbanschitsch, 1902; Zapparoli \& Reatto, 1969; see Soto-Faraco, Kingstone, \& Spence, 2003, and SotoFaraco \& Kingstone, 2004, for reviews), there has not, until very recently at least, been any systematic effort to study how motion signals coming from different sensory modalities are combined during perception.

In recent years, however, several important characteristics regarding multisensory contributions to motion processing have been revealed (e.g., Hofbauer et al., 2004; Kitagawa \& Ichihara, 2002; Meyer \& Wuerger, 2001; Soto-Faraco, Lyons, Gazzaniga, Spence, \& Kingstone, 2002; Soto-Faraco, Spence, \& Kingstone, 2004; Vroomen $\&$ de Gelder, 2003). For example, as a consequence of multisensory interactions in motion processing, observers often experience the illusion that a sound and a light move in the same direction even when the two stimuli are physically moving in opposite directions (e.g., Soto-Faraco et al., 2002; Zapparoli \& Reatto, 1969). Moreover, this illusion seems to occur over and above any interactions between static cues regarding spatial location (as typically measured in studies of ventriloquism). Despite this dissociation between static and dynamic crossmodal interactions, multisensory integration processes involving motion information share several important 
characteristics with the static ventriloquist illusion and other multisensory phenomena. For example, both dynamic motion capture and static ventriloquism depend on the stimulation of the different senses at approximately the same time (in keeping with the general rules of multisensory integration; Spence \& Squire, 2003; Stein $\&$ Meredith, 1993). Both effects have been shown to involve interactions at a perceptual level of processing (Kitagawa \& Ichihara, 2002; Soto-Faraco, Kingstone \& Spence, 2004; Vroomen \& de Gelder, 2003), although it must be noted that postperceptual factors can no doubt also play a role (i.e., Meyer \& Wuerger, 2001). Finally, as in studies of the static audiovisual ventriloquism effect, the interactions between moving auditory and visual stimuli are characterized by a marked asymmetry, with auditory perception routinely being modulated by visual motion, but not vice versa (Kitagawa \& Ichihara, 2002; Soto-Faraco, Spence, \& Kingstone, 2004). This asymmetry is in agreement with the notion that the sensory modality that provides the most reliable information regarding a given attribute - in this case, vision with respect to stimulus location and motion direction-will receive the greatest weighting in constructing an integrated percept (see Ernst \& Banks, 2002; Freides, 1974; Welch, 1999; Welch, DuttonHurt, \& Warren, 1986; Welch \& Warren, 1986).

\section{Scope of the Present Study}

In contrast with static ventriloquism studies, one important limitation of current behavioral studies of multisensory motion has been their restriction to audiovisual interactions. In the present study, we extend previous findings regarding audiovisual interactions in motion processing to the case of audition and touch. Extrapolating the findings obtained with one combination of sensory modalities to another combination is not trivial, given the unique role that each sense plays in providing information about the environment (e.g., Hughes, 1999; Stein \& Meredith, 1993). Indeed, in contrast to the audiovisual case, where the precision of vision in motion perception is clearly superior to that of audition, no clear pattern of dominance between audition and touch in motion processing or spatial localization has been established (e.g., Caclin, Soto-Faraco, Kingstone, \& Spence, 2002). Moreover, the same physical type of energy is involved in the auditory and the tactile sensory systems (involving mechanical pressure), which, again, is suggestive of the idea that there may be a tighter, perhaps more symmetrical, coupling (or relationship) between these two senses than between audition and vision (e.g., see Gescheider, 1970).

By examining audiotactile interactions, we were also able to investigate the spatial frames of reference that are active during multisensory integration of motion. Indeed, tactile receptors in the hand can adopt a variety of positions with respect to the head/trunk. This opens up an interesting research question, since tactile spatial location (and therefore, tactile motion direction) can be encoded in a number of different frames of reference. For instance, during early processing, the spatial location of a tactile stimulus is encoded according to a somatotopic frame of reference (i.e., according to where on the skin surface it occurred; Burton \& Sinclair, 1996; Penfield \& Rasmussen, 1950). However, during later processing, the location of tactile stimuli can also be encoded in a more abstract frame of reference independent of hand position (for instance, the neurons that are responsive to motion direction in the premotor ventral cortex of the monkey use a head-centered frame of reference; Graziano \& Gandhi, 2000; Graziano, Gross, Taylor, \& Moore, 2004). Adopting an abstract (i.e., external) frame of reference involves a remapping of tactile spatial information that is enabled by the integration of tactile inputs with proprioceptive and visual cues (e.g., Aglioti, Smania, \& Peru, 1999; Driver \& Grossenbacher, 1996; Lakatos \& Shepard, 1997b; Moscovitch \& Behrmann, 1994; Rinker \& Craig, 1994; Soto-Faraco, Ronald, \& Spence, in press; see Lloyd, Shore, Spence, \& Calvert, 2003, for recent neuroimaging evidence). Despite the need for this extra computational step, encoding tactile spatial information in an abstract frame of reference may be more useful in terms of manipulating and localizing objects in the environment. Furthermore, an abstract frame of reference would offer a natural common spatial framework to coordinate tactile stimuli with inputs from other sensory modalities, such as audition (see Lloyd, Merat, McGlone, $\&$ Spence, 2003) or vision (see Gray \& Tan, 2002; Kennett, Eimer, Spence, \& Driver, 2001).

Several researchers have recently shown, for example, that cross-modal links in spatial attention are updated as new body positions are adopted (see Lloyd, Merat, et al., 2003, and Eimer, Cockburn, Smedley, \& Driver, 2001, for the specific cases of touch and audition). Their results usually have shown that cross-modal attention operates in an external frame of reference in which the representation of object positions are remapped following posture change. Spatial remapping, however, does not occur under all circumstances. For example, Röder, Spence, and Rösler (2000) found that the attentional phenomenon of tactile inhibition of return (IOR) seems to operate in a somatotopically based frame of reference. Moreover, some studies of tactile spatial attention that have manipulated the congruency between the directions of motion of targets and distractors presented at different finger pads also have suggested that a somatosensory frame of reference may be prevalent under at least certain conditions (Evans \& Craig, 1991; Evans, Craig, \& Rinker, 1992). This gives rise to the question of whether the spatial representation of touch is updated according to body posture during the processing of multisensory motion information.

\section{EXPERIMENT 1}

The goal of the first experiment was to extend the cross-modal dynamic capture effect to a novel combination of sensory modalities-audition and touch. Ac- 
cording to several sources of evidence, one might expect to find some modulation of dynamic cues across audition and touch. One source of evidence comes from neurophysiological (Bremmer, Schlack, Duhamel, Graf, \& Fink, 2001) and neuroimaging (Bremmer, Schlack, Shah, et al., 2001a) studies, where the existence of brain areas subserving the common processing of somatosensory and auditory motion information has been strongly suggested (see Soto-Faraco et al., 2003, for a recent review). Another source of evidence comes from the study of intersensory conflict with spatially static inputs, where tactile stimuli have been shown to attract the perceived location of sounds (e.g., Caclin et al., 2002; Freedman \& Wilson, 1967; Pick, Warren, \& Hay, 1969).

We adapted the method developed by Soto-Faraco et al. (2002; Soto-Faraco, Spence, \& Kingstone, 2004) to study audiovisual integration of motion signals for the study of audio-tactile integration of motion. In this paradigm, two apparent motion streams (one in each sensory modality) are presented in each trial; they can move either in the same direction or in opposite directions. The observers are required to judge the direction of motion in one of the modalities (target modality) while trying to ignore the stimuli presented in the other modality (distractor modality). In half of the trials, the two motion streams are presented in synchrony, whereas in the other half the distractor stream is presented $500 \mathrm{msec}$ after the onset of the target stream. Here, we examined both potential combinations of target and distractor modalities in a blocked design, thereby reproducing the standard focused attention conditions used previously with this paradigm.

\section{Method}

Participants. Twelve undergraduate students from the University of British Columbia participated in this experiment in exchange for course credit. All had normal hearing and tactile sensitivity at the fingertips, and all were naive as to the purpose of the experiment.

Apparatus. Two loudspeaker cones (Audax VE100AO) were placed $30 \mathrm{~cm}$ from each other (center to center; see Figure 1A). Each loudspeaker cone was connected directly to each channel (left and right) of the computer's soundcard (ProAudio Basic 16, Media Vision). A vibrotactile stimulator (bone conduction vibrator, OticonA, $100 \Omega$ ) was mounted on one corner of each of the two foam cubes (7-cm side) placed directly in front of the loudspeaker cones. Two additional vibrotactile stimulators were placed beside each foam cube and served to mask any possible noise made by the target vibrators (see below). A red light-emitting diode (LED) was placed directly between the two loudspeakers to serve as a central fixation point. The inputs to the LED $(+5 \mathrm{v})$ and to the vibrators $(\mathrm{a} 200-\mathrm{Hz}$ wave generated from a BK Precision 3010 function generator, Dynascan Corp.) were controlled by relays operated from the parallel port of the computer. Two footpedals attached to the floor directly beneath the participants' left and right feet were used to collect responses. The Expe6 programming language (Pallier, Dupoux, \& Jeannin, 1997) was used to run the experimental protocol and to register all responses.

Materials. With this setup, apparent motion streams could be generated in audition and touch. ${ }^{1}$ Auditory apparent motion streams consisted of the presentation of two 50 -msec tones $(65 \mathrm{~dB}(\mathrm{~A})$ as measured from the participants' approximate head height, 5-msec amplitude envelope) in alternation to each loudspeaker cone, with an interstimulus interval (ISI) of $100 \mathrm{msec}$. The two tones used in a given apparent motion stream were of the same frequency, which was chosen randomly on a trial-by-trial basis from three possible frequencies: 450,500, and $550 \mathrm{~Hz}$ (this was done to discourage the participants from attempting to pick up some slight acoustic difference between the loudspeakers as a cue to the direction in which the auditory streams moved). Vibrotactile apparent motion streams consisted of two 50 -msec vibrations $(220 \mathrm{~Hz})$, one to each index finger, again with an ISI of $100 \mathrm{msec}$. The two additional vibrators (one on each side of the setup, far from the participant's skin) were activated at the same time as that at which either of the two vibrators in contact with the participants' fingers was switched on. This made any faint noise produced by the vibrations indistinguishable on each side of the setup, regardless of the particular finger vibrated. ${ }^{2}$

Procedure. The participants sat in front of the two loudspeakers at a distance of $40 \mathrm{~cm}$ and were instructed to hold the foam cubes (right cube with right hand, left cube with left hand), with their index fingers resting on the vibrotactile stimulators (see Figure 1A) and their feet pressed down on the footpedals. The experimenter encouraged the participants to keep their gaze fixed on the center of the setup. The room remained dark throughout the experiment.

The experiment was divided into two blocks of trials. In one block, the target modality was audition, and the distractor modality was touch, whereas in the other block the target and the distractor modalities were reversed. The order of presentation of these blocks was counterbalanced across participants. All trials consisted of the presentation of two apparent motion displays - one in the target modality and the other in the distractor modality. Each trial started with a 1,500-msec delay prior to the onset of the apparent motion stimuli in the target modality. As is illustrated in Figure 1B, the apparent motion stream in the distractor modality could be presented at either the same time as the apparent motion in the target modality (synchronous) or else $500 \mathrm{msec}$ later (asynchronous) and in either the same direction (congruent) or the opposite direction (conflicting). A total of 48 trials were presented in each of the two blocks, and the particular type of trial (synchronous-congruent, synchronous-conflicting, asynchronous-congruent, and asynchronous-conflicting) was randomly selected and equiprobable on every trial.

The participants were instructed to lift the footpedal on the side corresponding to the perceived direction of motion (left or right) in the target modality, while ignoring stimulation in the irrelevant distractor modality. The participants were told to respond accurately and not to worry about the speed of their responses. In fact, responses were not registered until at least $750 \mathrm{msec}$ after the auditory stimulus onset for all the conditions (a centrally located red LED was illuminated to signal that a response had been registered). The participants received a 10 -trial training session prior to the start of each experimental block. Training consisted of a direction-ofmotion discrimination task in the target modality (according to the block they were about to start) in the absence of any distractor stimuli. Training served to ensure that the participants understood the task and that the stimulation was well above threshold $(90 \%$ or higher accuracy in the training block was required to start the experimental block in each modality, and all the participants reached this criterion on their first attempt).

\section{Results and Discussion}

The accuracy data (proportion of correct responses) were submitted to an analysis of variance (ANOVA) with target/distractor combination (auditory target and tactile distractor vs. tactile target and auditory distractor), synchrony of presentation (synchronous vs. asynchronous), and congruency (congruent vs. conflicting) as withinsubjects factors. ${ }^{3}$ The analyses revealed a significant three-way interaction between block type, synchrony, and congruency $[F(1,11)=5.6, p<.05]$, and therefore, 


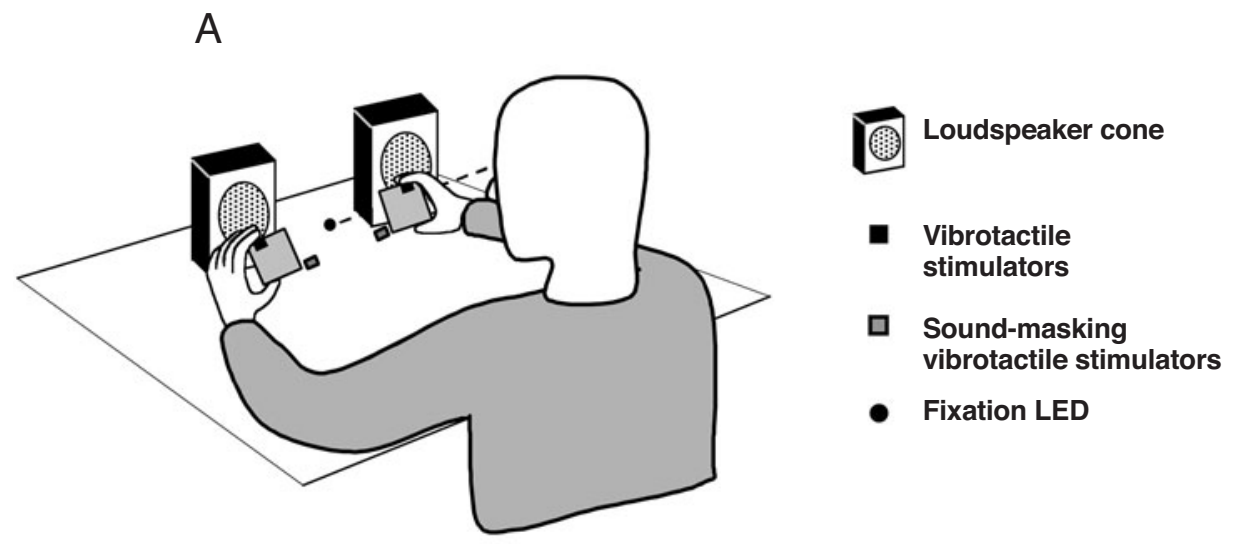

B

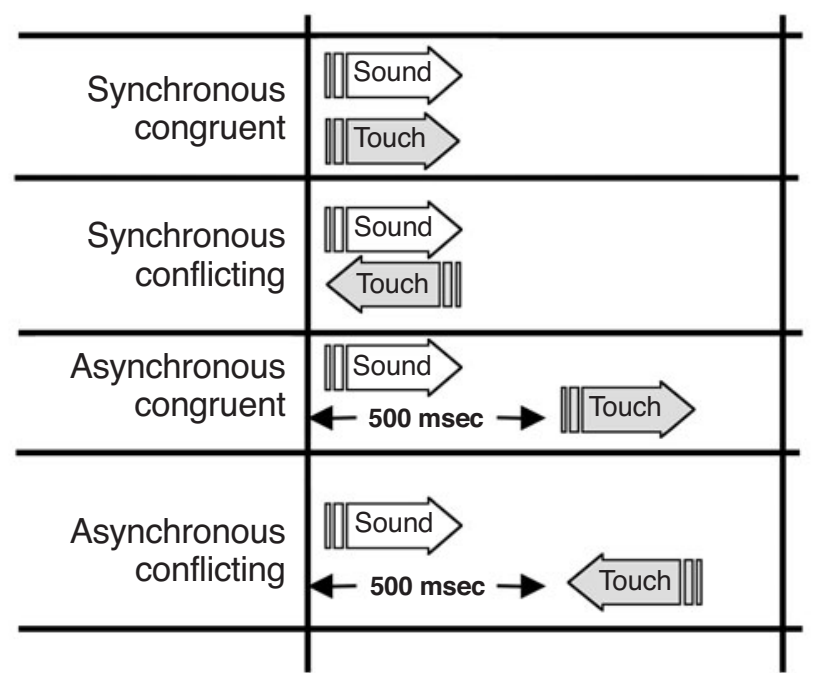

Figure 1. (A) Schematic representation of the experimental layout used in Experiment 1. The participant held two foam cubes, each incorporating one vibrotactile stimulator contacting the index finger, placed in front of the two loudspeaker cones. One light-emitting diode (LED) was placed at the center of the setup to serve as a fixation point. Unspeeded responses were made by the participant's momentarily lifting the right foot off one of two pedals under the toe and the heel (not shown). A 50-msec vibration was presented over the two masking vibrotactile stimulators in coincidence with the vibration of either of the two target stimulators, so the noise would not provide any spatial cue. (B) Example of the types of trials included in the auditory block of Experiment 1. In the tactile block, the auditory distractors could occur either simultaneously or delayed by 500 msec with respect to the vibrotactile targets.

we broke down the analyses by block type. When auditory apparent motion was relevant and touch irrelevant (see Figure 2A), the interaction between synchrony and congruency was significant $[F(1,11)=15.7, p<.005]$. This interaction was caused by a significant reduction in performance in conflicting trials, as compared with congruent trials, in the synchronous condition $[37 \%$ difference; $t(11)=4.4, p<.001]$, but not in the asynchronous condition $(0 \%$ difference; $|t|<1)$. Finally, in the block where tactile apparent motion was relevant and sounds were irrelevant (see Figure 2B), the interaction between congruency and synchrony also reached signif- icance $[F(1,11)=20.1, p=.001]$. The interaction was caused by a significant congruency effect in the synchronous condition $[14 \% ; t(11)=3.5, p=.005]$, but not in the asynchronous condition $(-0.05 \% ;|t|<1)$. Thus, in this experiment, congruency effects were reported for both target/distractor combinations in the synchronous trials. The interpretation of the significant three-way interaction between block type, synchrony, and congruency is, therefore, that the congruency effect shown in the synchronous trials was bigger for auditory motion targets and tactile distractors than for tactile motion targets with auditory distractors. 


\section{A. Auditory target / tactile distractor}

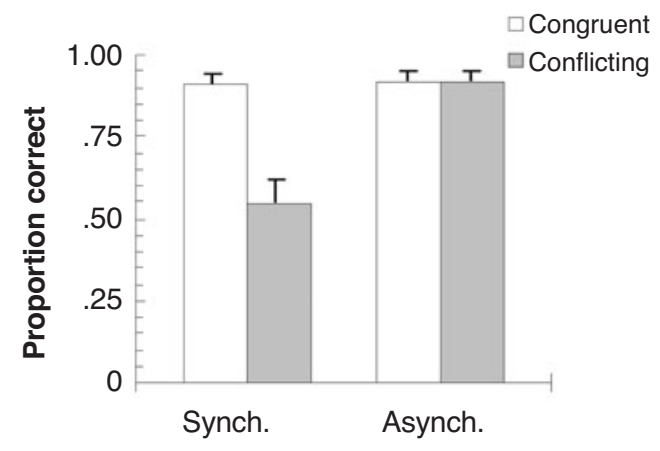

Synchrony

\section{B. Tactile target / auditory distractor}

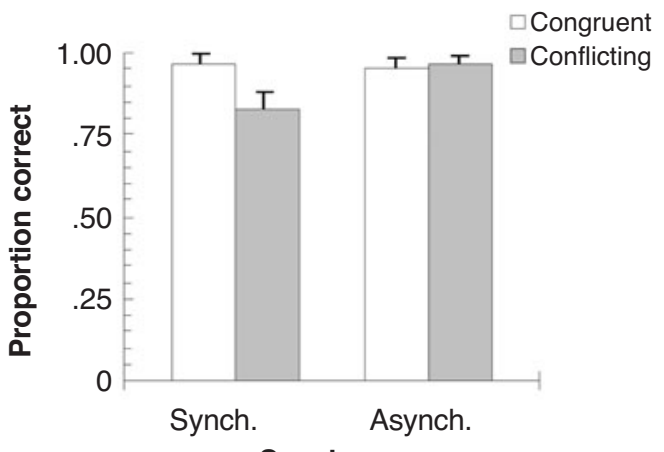

Synchrony

Figure 2. Graphs showing the mean accuracy (and standard error) in Experiment 1. Data are shown as a function of the target and distractor modalities, for each synchrony and congruency combination.

The main conclusion to emerge from Experiment 1 is that the cross-modal integration of dynamic information reported previously for audiovisual cues can generalize to other modality combinations. In particular, the present data suggest that the perceived direction of auditory apparent motion can also be strongly modulated by concurrent task-irrelevant tactile apparent motion. In addition, our results show that tactile apparent motion can be modulated by concurrent auditory motion, albeit to a lesser extent. Critically, no congruency effects were detected (and response accuracy was nearly perfect) in both modality combinations tested when irrelevant motion occurred asynchronously with the target motion. This result in the asynchronous trials provided a baseline performance measure for the motion discrimination task in the target modality, showing that perception of motion direction was unambiguous when the distractor motion was presented at a lag that minimized any possibility of multisensory integration with the target motion (e.g., Soto-Faraco et al., 2002). In addition, performance accuracy in the baseline condition confirmed that the participants' poor performance in the conflicting synchronous condition did not reflect merely confusion about which sensory modality they had to respond to in each block.

\section{EXPERIMENT 2}

As was discussed in the introduction, the sense of touch presents a particularly intriguing case for research on the spatial aspects of perception, because tactile motion information can be represented according to a number of different spatial frames of reference. Therefore, one important research question concerns the reference frame (or frames) relevant for the mechanisms of dynamic audiotactile integration. In Experiment 2, we examined whether the spatial frame of reference for tactile motion perception is represented in somatotopic ${ }^{4}$ coordinates or in a more abstract coordinate system involv- ing an externally based representation of space. We assessed auditory motion direction discrimination as a function of tactile distractors presented while the participants adopted one of two different body postures. Note that touch was irrelevant for the task, and therefore, the effects of body posture on tactile motion representation were assessed indirectly, through their modulation of auditory motion perception.

In the arms-uncrossed condition, the participants held the foam cubes containing the distractor vibrotactile stimulators exactly as in Experiment 1 . In this situation, the direction of motion coincides in somatotopic and in external coordinates; that is, a stream consisting of a vibration to the left hand followed by a vibration to the right hand moves rightward between two skin locations, as well as across external space, from the participant's viewpoint. In the arms-crossed condition, the participants held the cubes so that the left hand would hold the cube on the right and the right hand would hold the cube on the left. In this situation, direction of motion is different across somatotopic and external reference frames. A tactile stream consisting of a vibration to the left hand followed by a vibration to the right hand still moves rightward in terms of skin surface (i.e., in somatotopic coordinates), but it has a leftward direction with respect to the head and trunk (i.e., in external coordinates).

If the relevant spatial frame of reference during the audiotactile integration of motion is based on somatotopic coordinates, the congruency effects of touch on auditory directional discrimination should reverse when the arms are crossed, as compared with when the arms are placed in an uncrossed posture. However, if the tactile motion stimulus is encoded in an external frame of reference, tactile motion direction should remain the same regardless of body posture and, therefore, the significant congruency effects of touch on auditory directional discrimination should be equivalent across the two body postures. 


\section{Method}

Participants. Eight new participants were tested in this experiment. All were naive as to the purpose of the experiment and were paid with course credit for their participation. All reported normal touch and hearing.

Apparatus, Materials, and Procedure. In the arms-uncrossed condition, the method was exactly the same as in the block with auditory targets and tactile distractors of Experiment 1. In the armscrossed condition, everything was equal to the arms-uncrossed condition, except that the participants held the left (or the right) foam cube with their right (or left) hand (respectively). Each participant was tested in both conditions, with the order of conditions counterbalanced. It is important to note that, as in Experiment 1, the participants performed the task in complete darkness and, therefore, they did not have any direct visual feedback concerning the position of their body during the experiment. ${ }^{5}$

\section{Results and Discussion}

We conducted an ANOVA with posture (arms crossed or uncrossed), synchrony, and congruency as within-subjects factors (see Figure 3 for the average results in each condition). Note that in this experiment, the congruency factor can be arbitrarily defined in terms of the direction of tactile stimuli across the anatomical position of the hands or in terms of the direction of tactile stimuli in external space. Here, we used the physical direction of motion in external space to define congruency. The ANOVA revealed an interaction involving all three main terms $[F(1,7)=22.4$, $p=.002]$. To understand the nature of this three-way interaction, the data were examined separately as a function of posture. When the participants held their arms in the uncrossed posture (thus, in exactly the same posture as that in Experiment 1), there was a main effect of synchrony $[F(1,7)=24.3, p=.002]$ and a main effect of congruency $[F(1,7)=14.2, p=.007]$. Crucially, there was also a significant interaction between synchrony and congruency $[F(1,7)=45.8, p<.001]$, due to a significant effect of congruency in synchronous trials $[41 \% ; t(7)=5.2$, $p=.001]$, but not in asynchronous trials $[-4 \% ; t(7)=1.5$, $p=.17]$. In contrast, the analysis of the arms-crossed con- dition revealed no significant main effects of synchrony $[F(1,7)=3.0, p=.13]$ or congruency $[F(1,7)=1.7, p=$ $.23]$, as well as no significant interaction $[F(1,7)=3.2$, $p=.12]$. Consistent with this lack of an interaction, simple main effect tests within synchronous and asynchronous trials separately confirmed that there were no significant congruency effects in either condition $[0.25 \%,|t|<1$, and $13 \%, t(7)=1.6, p=.145$, respectively]. Overall, accuracy in the arms-crossed condition was high $(M=88 \%$, $S D=18)$ and statistically equivalent $(|t|<1)$ to the overall level of accuracy seen in the arms-uncrossed condition $(M=86 \%, S D=21)$.

The results in the arms-uncrossed condition replicated the tactile capture of auditory motion already found in Experiment 1, thus demonstrating the robustness of the effect. However, the critical result in this experiment was that adopting a crossed-arms posture eliminated the tactile dynamic capture effect on the discrimination of auditory motion direction. The implications of this result will be considered in detail below.

\section{GENERAL DISCUSSION}

The present study provided a behavioral test of multisensory integration of motion involving auditory and tactile information. The primary outcome is the unequivocal finding that motion cues in these two sensory modalities are integrated, producing a behavioral correlate that was measured here in terms of the cross-modal dynamic capture effect - namely, when motion cues in audition and touch contain directionally conflicting information, the correct discrimination of the target motion direction decreases dramatically for each of the modalities. Hence, this study demonstrated that audiotactile interactions in motion processing occur in both directions. That is, auditory motion has an influence on tactile motion processing, and vice versa. Finally, our present investigation did not reveal any clear-cut evidence for one particular type

\section{A. Arms uncrossed}

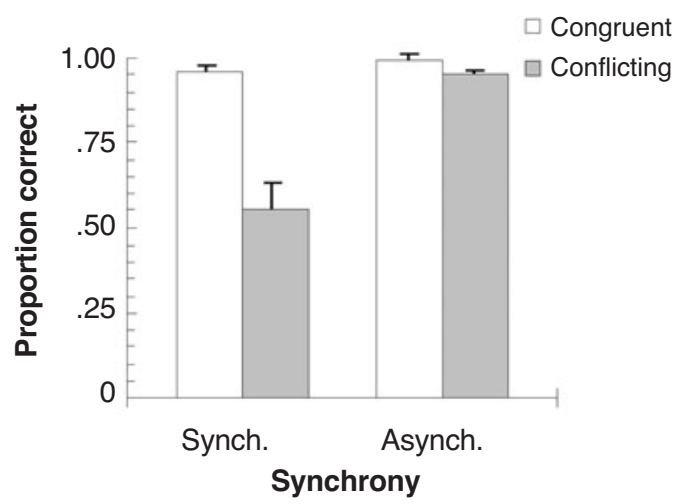

B. Arms crossed

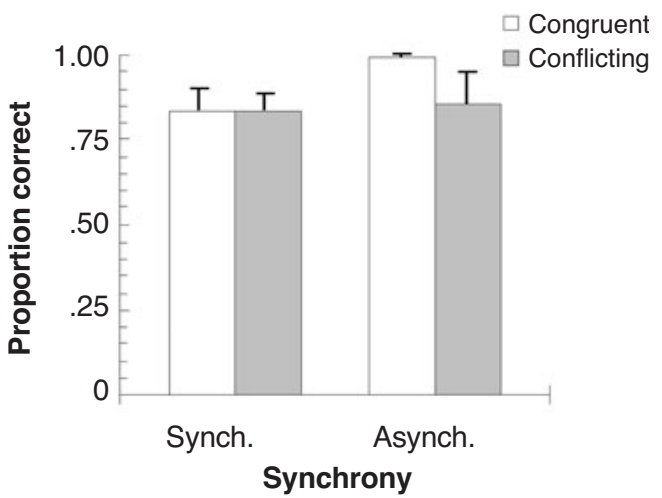

Figure 3. Graphs showing the mean accuracy (and standard error) in Experiment 2. Data are shown for each synchrony and congruency combination as a function of the different postures adopted: arms uncrossed (A) and arms crossed (B). 
of spatial frame of reference in which tactile motion direction is represented during multisensory integrationpurely somatotopic or purely externally defined.

\section{Cross-modal Asymmetries}

One interesting aspect of the present audiotactile results is that the cross-modal dynamic capture effect occurred for both modalities. This contrasts with the case of audition and vision, where auditory cues do not usually exert any significant influence on visual motion discrimination (Soto-Faraco, Kingstone, \& Spence, 2004; Soto-Faraco, Spence, \& Kingstone, 2004; although see Soto-Faraco et al., 2003, for an exception when the attentional load is high). This finding is important because it supports a view of multisensory integration of motion that is not based on the complete dominance of one sensory modality over the other, a process whereby the critical attribute of the "captured" modality would simply be replaced by the value of that attribute in the more dominant modality (Rock \& Harris, 1967; Rock \& Victor, 1963). As has been argued by several other researchers (e.g., Bertelson, 1999; de Gelder \& Bertelson, 2003; Ernst \& Banks, 2002; Welch, 1999), multisensory interactions are the result of integrative processes whereby the information coming from each sensory modality is weighted during perception. The present data strongly support and extend this argument to the multisensory integration of motion signals.

Another point of interest regards the nature of the cross-modal asymmetry observed, whereby tactile motion had a stronger effect on auditory motion perception than vice versa. A similar asymmetric pattern between audition and touch has been reported previously with other tasks, such as localization judgments of static stimuli (e.g., Fisher, 1962; Pick et al., 1969) and temporal order judgments (TOJs; e.g., Sherrick, 1976). For instance, Pick et al. found that vision exerted the strongest effect on touch and audition, whereas no noticeable effect of audition or touch was found on visual localization performance. Interestingly, just as in the present Experiment 1 , Pick et al. found that touch and audition exerted a reciprocal influence upon each other, with an imbalance in favor of touch (i.e., the effects of touch on audition were bigger than in the reverse condition; see Fisher, 1962, for similar results). In another study, Sherrick tested cross-modal interactions in TOJs where the stimuli in the target modality (two tactile events or two sounds) were presented in succession at different locations (left or right). Two stimuli in the irrelevant modality (sounds or touches, respectively) were presented concurrently in the same or the opposite order of spatial locations. Over-all, Sherrick's results showed that performance was less accurate when the order of stimuli in the irrelevant modality conflicted with that of the order of the target stimuli, with a stronger effect of touch on audition than vice versa. ${ }^{6}$

The close coincidence in the dominance pattern for audiotactile inputs across very different methodologies and materials (e.g., Fisher, 1962; Pick et al., 1969; Sher- rick, 1976; Experiment 1, in the present study) suggests that it may have, at least partially, a basis in processing features intrinsic to the perceptual system. As is the case for the audiovisual results, however, extrapolating this putative pattern of dominance beyond the framework of each particular study must be done with some caution. For instance, one could argue that if other intensity values had been chosen - for instance, louder sounds and less intense vibrotactile stimulation - the hierarchy of dominance would have changed. Note, though, that such an argument would not invalidate in any way our primary findings regarding the existence of reciprocal crossmodal interactions between touch and audition.

\section{Spatial Frame of Reference}

Another issue addressed in the present study is the spatial frame of reference in which tactile motion direction is represented during multisensory integration. This question arises naturally when the sense of touch is evaluated in a spatial task (e.g., Driver \& Grossenbacher, 1996; Soto-Faraco et al., in press), because the direction/ location of tactile stimuli can be encoded according to a somatosensory-based or an externally based frame of reference. As was noted above, the results obtained in Experiment 2 were unexpected because the consequences of crossing the arms on the cross-modal dynamic capture effect did not conform fully to any of the two previously advanced outcomes, as one might have expected from the clear-cut results of some recent studies in which crossed versus uncrossed body postures were used in different tasks (see Röder et al., 2000, for evidence supporting a somatotopic frame of reference in inhibition of return; see Lloyd, Merat, et al., 2003, for evidence supporting an external frame of reference in endogenous spatial attention).

Intuitively, the most straightforward explanation of the result of Experiment 2 would be that crossing the arms abolishes cross-modal integration. Although this interpretation has the appeal of simplicity, there are now many demonstrations of cross-modal interactions occurring in several different postural manipulations (and, among them, the arms-crossed posture used here is probably the most popular; Lloyd, Merat, et al., 2003; see Spence \& Driver, 2004, for a recent review). Therefore, if the view that crossing the arms turns off multisensory interactions is to be maintained, one should then provide a reasonable causal explanation. One such explanation, consistent with the existing literature, may be that crossing the hands altered the reliability of the computations of tactile motion direction in our experiment, consequently diminishing its influence on the perception of auditory motion direction. In keeping with this idea, various recent findings have confirmed that people are often confused regarding the actual body location of touch when asked to localize two rapidly presented vibrotactile stimuli while their arms are crossed (Shore, Spry, \& Spence, 2002; Yamamoto \& Kitazawa, 2001; see also Benedetti, 1985, 1988, Ponzo, 1910, Spence, Harris, \& Zampini, 2003, and Tastevin, 1937, for other examples 
of tactile confusions as a consequence of crossing body parts across the midline).

As was discussed in the introduction, for the tactile input to be represented in an external frame of reference, a spatial remapping involving proprioceptive and/or visual cues must occur (e.g., Driver \& Grossenbacher, 1996; Kitazawa, 2002; Shore et al., 2002; Soto-Faraco et al., in press; Spence, Kingstone, Shore, \& Gazzaniga, 2001; Spence, Shore, Gazzaniga, Soto-Faraco, \& Kingstone, 2001). This leads to another possible interpretation of the finding that crossing the arms eliminated the tactile influence on the perception of auditory motion direction - namely, that only a partial spatial remapping of tactile input occurred. This may initially seem an unlikely hypothesis, since a remapping system that functions only partially would probably result in a loss of efficiency for the individual in most situations. However, there is now ample evidence that partial remapping in the brain may be more prevalent than one would initially think (e.g., Pouget, Deneve, \& Duhamel, 2002), both at a cortical (e.g., Cohen \& Andersen, 2000; Duhamel, Bremmer, BenHamed, \& Graf, 1997) and at a subcortical (Jay \& Sparks, 1987) level. The ubiquity of partially shifting receptive fields may, therefore, explain the partial remapping of touch, especially when the recalibration needed is large, as would be the case when the two arms are fully crossed. Indeed, results consistent with a partial remapping account have recently been found in other behavioral studies regarding touch and proprioception (see Röder, Rösler, \& Spence, 2004; Spence, Baddeley, Zampini, James, \& Shore, 2003).

\section{Concluding Remarks}

The present study has addressed, for the first time, the interactions in motion processing between audition and touch at a behavioral level. We used the cross-modal dynamic capture task (Soto-Faraco et al., 2002; Soto-Faraco, Spence, \& Kingstone, 2004) to reveal that apparent motion in one modality (touch or audition) affected the processing of apparent motion in another modality (audition or touch, respectively), in clear agreement with what would be expected from the results of neuroimaging (Bremmer, Schlack, Duhamel, et al., 2001; Hagen et al., 2002; Lewis, Beauchamp, \& DeYoe, 2000) and neurophysiological (Bremmer, Schlack, Shah, et al., 2001; Duhamel, Colby, \& Goldberg, 1998) studies revealing brain areas related to motion processing in more than one sensory modality. Two additional interesting aspects of motion interactions that are characteristic of the audiotactile case were also revealed in this research. One is that, contrary to what happens with audiovisual combinations, the multisensory interaction observed between touch and audition is reciprocal, although not equivalent in strength. It will be important to determine the degree to which this asymmetry reflects the organization of the perceptual system versus the particular set of stimulus parameters used. The other aspect is that crossing the arms across the body midline rendered the effects of tactile apparent motion distractors ineffective. It remains for further in- vestigation to determine whether this modulation of body posture should be attributed to a decrement in the ability of the tactile system to compute motion direction with the arms-crossed posture.

Finally, it is important to note that, just as happened after the first reports of the audiovisual version of the dynamic capture effect, two general issues will need to be addressed in order to characterize this audiotactile version of the effect (see Soto-Faraco et al., 2003, for a review). One will be to determine whether motion capture plays a critical role over and above the potential location capture effect of each static event individually (i.e., the ventriloquist illusion). The second will be to determine the level(s) of processing at which the capture effect takes place - that is, whether this capture phenomenon reflects a perceptual process occurring automatically and before each individual motion signal is available to awareness, or whether it is the reflection of a more generic stimulusresponse interference enabled by the effects of the distractor motion on later output stages. If past research is to prove an accurate guide to these future investigations, it is our proposal that motion plays a critical role over and above the standard static ventriloquism effect.

\section{REFERENCES}

Aglioti, S., Smania, N., \& Peru, A. (1999). Frames of reference for mapping tactile stimuli in brain-damaged patients. Journal of Cognitive Neuroscience, 11, 67-79.

ALLEN, P. G., \& Kolers, P. A. (1981). Sensory specificity of apparent motion. Journal of Experimental Psychology: Human Perception \& Performance, 7, 1318-1326.

Benedetti, F. (1985). Processing of tactile spatial information with crossed fingers. Journal of Experimental Psychology: Human Perception \& Performance, 11, 517-525.

BenedeTti, F. (1988). Exploration of a rod with crossed fingers. Perception \& Psychophysics, 44, 281-284.

Benussi, V. (1913). Kinematohaptische Erscheinungen (Vorläufige Mitteilung über Scheinbewegungsauffassung aufgrund haptischer Eindrücke) [Kinematohaptic illusion (preliminary report on apparent motion from haptic sensations)]. Archiv für die Gesamte Psychologie, 29, 385-388.

Benussi, V. (1914). Kinematohaptische Scheinbewegungen und Auffassungsumformung [Kinematohaptic apparent movement and the change of perception]. In Bericht VI Kongress für Experimentelle Psychologie (pp. 31-35). Göttingen.

Bertelson, P. (1999). Ventriloquism: A case of crossmodal perceptual grouping. In G. Aschersleben, T. Bachmann, \& J. Müsseler (Eds.), Cognitive contributions to the perception of spatial and temporal events (pp. 347-362). Amsterdam: Elsevier.

Bertelson, P., \& DE Gelder, B. (2004). The psychology of multimodal perception. In C. Spence \& J. Driver (Eds.), Crossmodal space and crossmodal attention (pp. 141-177). Oxford: Oxford University Press.

Bremmer, F., Schlack, A., Duhamel, J. R., Graf, W., \& Fink, G. R. (2001). Space coding in primate parietal cortex. NeuroImage, 14, S46-S51.

Bremmer, F., Schlack, A., Shah, N. J., Kubischik, M., Hoffman, K. P., Zilles, K., \& FinK, G. R. (2001). Polymodal motion processing in posterior parietal and premotor cortex: A human fMRI study strongly implies equivalencies between humans and monkeys. $\mathrm{Neu}$ ron, 29, 287-296.

BURTON, H., \& Sinclair, R. (1996). Somatosensory cortex and tactile perceptions. In L. Kruger (Ed.), Pain and touch (pp. 105-177). San Diego: Academic Press. 
Caclin, A., Soto-Faraco, S., Kingstone, A., \& Spence, C. (2002). Tactile "capture" of audition. Perception \& Psychophysics, 64, 616630.

Cohen, Y. E., \& Andersen, R. A. (2000). Reaches to sounds encoded in an eye-centered reference frame. Neuron, $\mathbf{3}, 647-652$.

DE Gelder, B., \& BERTELSON, P. (2003). Multisensory integration, perception and ecological validity. Trends in Cognitive Sciences, 7, 460467.

Driver, J., \& Grossenbacher, P. G. (1996). Multimodal spatial constraints on tactile selective attention. In T. Inui \& J. L. McClelland (Eds.), Attention and performance XVI: Information integration in perception and communication (pp. 209-235). Cambridge, MA: MIT Press.

Duhamel, J. R., Bremmer, F., BenHamed, S., \& Graf, W. (1997). Spatial invariance of visual receptive fields in parietal cortex neurons. Nature, 389, 845-848.

Duhamel, J. R., Colby, C. L., \& GoldberG, M. E. (1998). Ventral intraparietal area of the macaque: Congruent visual and somatic response properties. Journal of Neurophysiology, 79, 126-136.

Eimer, M., Cockburn, D., Smedley, B., \& Driver, J. (2001). Crossmodal links in endogenous spatial attention are mediated by common external locations: Evidence from event-related brain potentials. Experimental Brain Research, 139, 398-411.

ERnst, M. O., \& BANKS, M. S. (2002). Humans integrate visual and haptic information in a statistically optimal fashion. Nature, $\mathbf{4 1 5}$, 429-433.

Evans, P. M., \& Craig, J. C. (1991). Tactile attention and the perception of moving tactile stimuli. Perception \& Psychophysics, 49, 355364.

Evans, P. M., Craig, J. C., \& Rinker, M. A. (1992). Perceptual processing of adjacent and nonadjacent tactile nontargets. Perception \& Psychophysics, 52, 571-581.

FISHER, G. H. (1962). Resolution of spatial conflict. Bulletin of the British Psychological Society, 46, 3A.

Freedman, S. J., \& Wilson, L. (1967). Compensation for auditory rearrangement following exposure to auditory-tactile discordance. Perceptual \& Motor Skills, 25, 861-866.

FREIDES, D. (1974). Human information processing and sensory modality: Cross-modal functions, information complexity, memory, and deficit. Psychological Bulletin, 81, 284-310.

GESCHEIDER, G. A. (1970). Some comparisons between touch and hearing. IEEE Transactions on Man-Machine Systems, 11, 28-35.

GraY, R., \& TAN, H. Z. (2002). Dynamic and predictive links between touch and vision. Experimental Brain Research, 145, 50-55.

GraZiano, M. S. A., \& GaNDHI, S. (2000). Location of the polysensory zone in the precentral gyrus of anesthetized monkeys. Experimental Brain Research, 135, 259-266.

Graziano, M. S. A., Gross, C. G., Taylor, C. S. R., \& Moore, T. (2004). A system of multimodal areas in the primate brain. In C. Spence \& J. Driver (Eds.), Crossmodal space and crossmodal attention (pp. 51-67). Oxford: Oxford University Press.

Hagen, M. C., Franzen, O., McGlone, F., Essick, G., Dancer, C., \& PARDO, J. V. (2002). Tactile motion activates the human MT/V5 complex. European Journal of Neuroscience, 16, 957-964.

Hofbauer, M., Wuerger, S. M., Meyer, G. F., Roehrbein, M., Schill, K., \& Zetzsche, C. (2004). Catching audiovisual mice: Predicting the arrival time of auditory-visual motion signals. Cognitive, Affective, \& Behavioral Neuroscience, 4, 245-254.

Howard, I. P., \& Templeton, W. B. (1966). Human spatial orientation. New York: Wiley.

Hughes, H. C. (1999). Sensory exotica: A world beyond human experience. Cambridge, MA: MIT Press.

IWAMURA, Y., IRIKI, A., \& TANAKA, M. (1994). Bilateral hand representation in the postcentral somatosensory cortex. Nature, 369, 554-556.

JAY, M. F., \& SPARKS, D. L. (1987). Sensorimotor integration in the primate superior colliculus: I. Motor convergence. Journal of Neurophysiology, 57, 22-34.

KennetT, S., EIMer, M., Spence, C., \& Driver, J. (2001). Tactilevisual links in exogenous spatial attention under different postures: Convergent evidence from psychophysics and ERPs. Journal of $\mathrm{Cog}$ nitive Neuroscience, $\mathbf{1 3}, \underline{462-478 .}$
Kitagawa, N., \& Ichihara, S. (2002). Hearing visual motion in depth. Nature, 416, 172-174.

KiTAZAWA, S. (2002). Where conscious sensation takes place. Consciousness \& Cognition, 11, 475-477.

LAKATOS, S., \& ShEPARD, R. N. (1997a). Constraints common to apparent motion in visual, tactile and auditory space. Journal of Experimental Psychology: Human Perception \& Performance, 23, 1050-1060.

LaKatos, S., \& ShePard, R. N. (1997b). Time-distance relations in shifting attention between locations on one's body. Perception \& Psychophysics, 59, 557-566.

Lewis, J. W., BeAUChamp, M. S., \& DeYoe, E. A. (2000). A comparison of visual and auditory motion processing in human cerebral cortex. Cerebral Cortex, 10, 873-888.

Lloyd, D. M., Merat, N., McGlone, F., \& Spence, C. (2003). Crossmodal links between audition and touch in covert endogenous spatial attention. Perception \& Psychophysics, 65, 901-924.

Lloyd, D. M., Shore, D. I., Spence, C., \& Calvert, G. A. (2003). Multisensory representation of limb position in human premotor cortex. Nature Neuroscience, 6, 17-18.

Mateef, S., Hohnsbein, J., \& NoACK, T. (1985). Dynamic visual capture: Apparent auditory motion induced by a moving visual target. Perception, 14, 721-727.

MEYER, G. F., \& WUERGER, S. M. (2001). Cross-modal integration of auditory and visual motion signals. NeuroReport, 12, 2557-2560.

Moscovitch, M., \& Behrmann, M. (1994). Coding of spatial information in the somatosensory system: Evidence from patients with neglect following parietal lobe damage. Journal of Cognitive Neuroscience, 6, 151-155.

PaLlier, C., Dupoux, E., \& JeAnnin, X. (1997). EXPE: An expandable programming language for on-line psychological experiments. $B e-$ havior Research Methods, Instruments, \& Computers, 29, 322-3i27.

PENFIELD, W., \& Rasmussen, T. (1950). The cerebral cortex of man. New York: Macmillan.

Pick, H. L., JR., Warren, D. H., \& Hay, J. C. (1969). Sensory conflict in judgments of spatial direction. Perception \& Psychophysics, 6, 203-205.

Ponzo, M. (1910). Intorno ad alcune illusioni nel campo delle sensazioni tattili, sull'illusione di Aristotele e fenomeni analoghi [On some tactile illusions, Aristotle's illusion, and similar phenomena]. Archive für die Gesamte Psychologie, 16, 307-345.

Pouget, A., Deneve, S., \& Duhamel, J. R. (2002). A computational perspective on the neural basis of multisensory spatial representations. Nature Reviews Neuroscience, 3, 741-747.

Rinker, M. A., \& Craig, J. C. (1994). The effect of spatial orientation on the perception of moving tactile stimuli. Perception \& Psychophysics, 56, 356-362.

Rock, I., \& HARrIS, C. S. (1967). Vision and touch. Scientific American, 216, 96-104.

Rock, I., \& VICTOR, J. (1963). Vision and touch: An experimentally created conflict between the two senses. Science, 143, 594-596.

RöDER, B., Rösler, F., \& SPENCE, C. (2004). Early vision impairs tactile perception in the blind. Current Biology, 14, 121-124.

RöDER, B., SPENCE, C., \& RöSLER, F. (2000). Inhibition of return and oculomotor control in the blind. NeuroReport, 11, 3043-3045.

SHERrICK, C. E. (1976). The antagonisms of hearing and touch. In S. K. Hirsh, D. H. Eldredge, I. J. Hirsh, \& S. R. Silverman (Eds.), Hearing and Davis: Essays honoring Hallowell Davis (pp. 149-158). St. Louis: Washington University Press.

Shore, D. I., Spry, E., \& Spence, C. (2002). Confusing the mind by crossing the hands. Cognitive Brain Research, 14, 153-163.

Soto-Faraco, S., \& KingSTONE, A. (2004). Multisensory integration of dynamic information. In G. Calvert, C. Spence, \& B. E. Stein (Eds.), The handbook of multisensory processes (pp. 49-67). Cambridge, MA: MIT Press.

Soto-Faraco, S., Kingstone, A., \& Spence, C. (2003). Multisensory contributions to the perception of motion. Neuropsychologia, $\mathbf{4 1}$, 1847-1862.

Soto-Faraco, S., Kingstone, A., \& Spence, C. (2004). Automatic visual capture of auditory motion. Manuscript submitted for publication.

Soto-Faraco, S., Lyons, J., Gazzaniga, M., Spence, C., \& KingSTONE, A. (2002). The ventriloquist in motion: Illusory capture of 
dynamic information across sensory modalities. Cognitive Brain Research, 14, 139-146.

Soto-Faraco, S., Ronald, A., \& Spence, C. (in press). Tactile selective attention and body posture: Assessing the multisensory contributions of vision and proprioception. Perception \& Psychophysics.

Soto-Faraco, S., Spence, C., \& Kingstone, A. (2004). Cross-modal dynamic capture: Congruency effects in the perception of motion across sensory modalities. Journal of Experimental Psychology: Human Perception \& Performance, 30, 330-345.

Spence, C., Baddeley, R., Zampini, M., James, R., \& Shore, D. I. (2003). Multisensory temporal order judgments: When two locations are better than one. Perception \& Psychophysics, 65, 318-328.

Spence, C., \& Driver, J. (Eds.), (2004). Crossmodal space and crossmodal attention. Oxford: Oxford University Press.

Spence, C., Harris, C., \& ZAmpini, M. (2003, July). The "Japanese illusion" revisited? Impaired vibrotactile movement discrimination with interleaved fingers. Paper presented at the EPS Meeting, Reading, UK.

Spence, C., Kingstone, A., Shore, D. I., \& Gazzaniga, M. S. (2001). Representation of visuotactile space in the split brain. Psychological Science, 12, 90-93.

Spence, C., Pavani, F., \& Driver, J. (2004). Spatial constraints on visual-tactile cross-modal distractor congruency effects. Cognitive, Affective, \& Behavioral Neuroscience, 4, 152-173.

Spence, C., Shore, D. I., Gazzaniga, M. S., Soto-Faraco, S., \& KingSTONE, A. (2001). Failure to remap visuotactile space across the midline in the split-brain. Canadian Journal of Experimental Psychology, 55, 135-142.

SPence, C., \& Squire, S. B. (2003). Multisensory integration: Maintaining the perception of synchrony. Current Biology, 13, R519-R521.

StaAl, H. E., \& Donderi, D. C. (1983). The effect of sounds on visual apparent movement. American Journal of Psychology, 96, 95-105.

Stein, B. E., \& Meredith, M. A. (1993). The merging of the senses. Cambridge, MA: MIT Press.

TAstevin, J. (1937). En partant de l'expérience d'Aristote: Les déplacements artificiels des parties du corps ne sont pas suivis par le sentiment de ces parties ni par les sensations qu'on peut y produire [Starting from Aristotle's illusion: The artificial displacements of parts of the body are not followed by feeling in these parts or by the sensations which can be produced there]. L'Encephale, 1, 57-84, 140-158.

URBANTSCHITSCH, V. (1902). Über die Beeinflussung subjektiver Gesichtsempfindungen [On the manipulation of subjective sensations at the face]. Pflügers Archiv für die geschichte Physiologie, 94, 347-448.

Vroomen, J., \& DE Gelder, B. (2003). Visual motion influences the contingent auditory motion aftereffect. Psychological Science, $\mathbf{1 4}_{2}$ 357-361.

WERTHEIMER, M. (1912). Experimentelle Studien über das Sehen von Bewegung [Experimental studies on the visual perception of movement]. Zeitschrift für Psychologie, 61, 161-265.

WelCH, R. B. (1999). Meaning, attention, and the "unity assumption" in the intersensory bias of spatial and temporal perceptions. In G. Aschersleben, T. Bachmann, \& J. Musseler (Eds.), Cognitive contributions to the perception of spatial and temporal events (pp. 371-387). Amsterdam: Elsevier.

Welch, R. B., DuttonHurt, L. D., \& Warren, D. H. (1986). Contributions of audition and vision to temporal rate perception. Perception \& Psychophysics, 39, 294-300.

WELCH R. B., \& WARREN D. H. (1986). Intersensory interactions. In K. R. Boff, L. Kaufman, \& J. P. Thomas (Eds.), Handbook of perception and human performance: Vol. 1, Sensory processes and perception (pp. 25.1-25.36). New York: Wiley.
Whitsel, B. L., Roppolo, J. R., \& Werner, G. (1972). Cortical information processing of stimulus motion on primate skin. Journal of Neurophysiology, 35, 691-717.

YAMAMOTO, S., \& KiTAZAWA, S. (2001). Reversal of subjective temporal order due to arm crossing. Nature Neuroscience, 4, 759-765.

Zapparoli, G. C., \& Reatto, L. L. (1969). The apparent movement between visual and acoustic stimulus and the problem of intermodal relations. Acta Psychologica, 29, 256-267.

\section{NOTES}

1. The experience of tactile apparent motion was initially documented by Benussi $(1913,1914)$, shortly after the original Wertheimer (1912) paper on visual apparent motion. Since then, several researchers have confirmed and extended the effect. Moreover, it seems that the constraints for the experience of apparent motion may follow the same principles in vision, audition, and touch (e.g., Lakatos \& Shepard, 1997a).

2. The noise produced by the additional vibrators was substantially more intense than the noise produced by the experimental vibrators, given that they were in contact with a hard surface (the table top) that amplified their sound. The vibrators delivering the vibrotactile stimulation to the fingers rested on the foam cube, a material that helped to attenuate any noise that they made.

3. Preliminary data analysis revealed that the order in which the blocks were presented did not give rise to any significant main effects, nor was it involved in any significant interaction in any of the analyses (all $p \mathrm{~s}>.05$ ); therefore, we do not report this factor.

4. It must be noted that the hypothesis of a somatopic representation does not necessarily imply that these representations are to be based on the primary somatosensory cortex (SI). Indeed, this is an unlikely possibility, because SI does not contain bilateral representations, which are probably implicated in apparent motion perception across the two hands. There are, however, bilateral somatosensory representations as early as in the secondary somatosensory cortex (e.g., Iwamura, Iriki, \& Tanaka, 1994), an area that has also been shown to contain directionsensitive neurons in animal studies (see Whitsel, Roppolo, \& Werner, 1972).

5. In an attempt to evaluate the influence of direct visual feedback on the postural modulations, we performed two pilot manipulations. One was similar to Experiment 2 in the relevant details, including the fact that the participants were tested with the room lights switched off. The other pilot manipulation was the same, except that the room lights remained switched on during the experiment. No statistical differences were found between the two groups, or when compared with the results of Experiment 2 in terms of the pattern of interactions found. This suggests that the potential influence of direct visual feedback on body posture was minimal and, therefore, supports the argument that the results found here are due mostly to proprioceptive influences on tactile remapping (see Spence, Pavani, \& Driver, 2004, for similar findings from a cross-modal congruency task).

6. From visual inspection of Sherrick's data (Sherrick, 1976, see the graphs on pp. 153-154), it appears that auditory TOJs were influenced more by incongruent tactile stimuli (a drop in accuracy of approximately $20 \%-30 \%$ ) than tactile TOJs were influenced by incongruent sounds (a drop in accuracy of $4 \%-10 \%$ ). This result once again resembles the audiotactile asymmetry reported in the present study.

(Manuscript received October 15, 2002; revision accepted for publication April 30, 2004.) 